\title{
A REVIEW OF MODELLING APPROACHES FOR FLOW AND HEAT TRANSFER IN NANOFLUIDS
}

\author{
JURE RAVNIK \& JAN TIBAUT \\ Faculty of Mechanical Engineering, University of Maribor, Slovenia
}

\begin{abstract}
When designing devices in the field of process, power and heat engineering the choice of the fluid that transports heat, mass and momentum is crucial. The thermal properties of such a fluid defines the efficiency of the device. Since the thermal properties of the standard heat transfer fluids, such as water or oil, are not optimal, nanofluids were introduced. A nanofluid is a term describing a dilute dispersion of particles in a fluid. The diameter of particles is in the order of ten nanometres. The particles are made of metal oxides, which enhance the thermal properties of the suspension. In this paper we will present the current trends in nanofluid modelling - from the effective properties approach, an approach that features additional equation for nanofluid concentration - to Euler-Lagrange type approaches.
\end{abstract}

Keywords: nanofluid, suspension, modelling, computational fluid dynamics.

\section{INTRODUCTION}

Cooling and heating are demanded in many engineering applications. In order to efficiently disperse or transport heat, the introduction of nanoparticles into a fluid was suggested by Choi [1]. The particles are nanometre-sized $(10-50 \mathrm{~nm})$ and have approximately uniform distribution in the base fluid. Such a suspension is called nanofluid and has improved thermal properties compared to base fluid (Yang et al. [2], Minea [3]).

Several techniques for nanoparticle preparation and dispersion have been proposed (Wang and Mujumdar [4]). Many authors have focused on experimental and numerical investigation of natural convection of nanofluids [5]-[16]. Several authors studied forced and mixed convection as well. Among numerical studies [17]-[20] the lid-driven cavity and the differentially heated cavity are most researched. Many experimental works have also been published, [21], where turbulent as well as laminar flow regimes are considered.

In terms of the numerical solution of the governing equations, computational fluid dynamics software can be used to simulate heat transfer and flow of nanofluids. Research into finding efficient new numerical methods is currently ongoing, with finite volume method [22] being the most promising candidate. Other approaches include control volume based finite element method [23], the lattice Boltzmann method [24], the finite difference method [25], [26], and the boundary element method [27].

There seems not to be a general consensus on the best model to describe nanofluid behaviour. In this paper, we will review the most common nanofluid modelling approaches and summarize best practices. Due to the fact that the nanofluid is a stable suspension the simplest model is to assume that the nanoparticle concentration is uniform throughout the suspension. With the assumption of uniform particle distribution and due to good thermal properties of the nanoparticle material, we may assume that the particles are in thermal equilibrium with the fluid (the Biot number value is very small). In this case, the simplest model of nanofluid is to consider the nanofluid as a fluid with changed properties and use models to evaluate these properties based on the fluid temperature or nanoparticle volume fraction. A more advanced idea is to model the nanofluid concentration with an additional transport equation, which enables us to capture the effect of thermophoresis and Brownian diffusion. Finally, Euler-Lagrange type approaches are possible, where nanoparticles (or 
groups of nanoparticles) are tracked within the fluid and thus interaction with the fluid is achieved through a one-way coupling.

\section{MODELLING}

\subsection{Flow equations}

Nanofluid is a suspension, which uses a base fluid, which is usually water, glycol or oil. Most base fluids are incompressible, thus the assumption of incompressibility of a nanofluid is reasonable. Density variations are taken into account only in the buoyancy term when natural convection play in important role. We also assume no chemical reactions, no external body forces apart from gravity, a dilute mixture, no viscous dissipation and no radiative heat transfer. We assume that the base fluid and the particles are in thermal equilibrium.

Let $\vec{u}$ be the flow velocity. In this case the continuity equation reads

$$
\nabla \cdot \vec{u}=0
$$

The Navier-Stokes equation, which governs the transport of momentum, can be written as

$$
\rho \frac{\partial \vec{u}}{\partial t}+\rho(\vec{u} \cdot \nabla) \vec{u}=\rho \vec{g}-\nabla p+\nabla \cdot \underline{\tau},
$$

where $t$ is time, $\rho$ density, $p$ pressure and $\vec{g}$ gravitational acceleration. The viscous stress tensor $\underline{\tau}$ may be for a Newtonian fluid expressed as $\underline{\tau}=2 \mu \underline{\dot{\epsilon}}=\mu\left(\nabla \vec{u}+(\nabla \vec{u})^{t}\right)$. The energy transport equation for the considered fluid is

$$
\frac{\partial\left(\rho c_{p} T\right)}{\partial t}+\nabla \cdot\left(\rho c_{p} T \vec{u}\right)=\nabla \cdot(k \nabla T)+S_{e}
$$

where $c_{p}$ is the heat capacity and $k$ the thermal conductivity.

An alternative formulation of governing equations may be derived by using the curl operator on the Navier-Stokes equation. We obtain the velocity-vorticity formulation of the Navier-Stokes equations. The velocity-vorticity formulation includes the kinematics equation and the vorticity transport equation. The kinematics equation can be stated for an incompressible fluid (Ravnik [28]) in the following way:

$$
\nabla^{2} \vec{u}+\nabla \times \vec{\omega}=
$$

and the vorticity transport equation is

$$
\begin{aligned}
\frac{\partial \vec{\omega}}{\partial t}+(\vec{u} \cdot \nabla) \vec{\omega}=(\vec{\omega} \cdot \nabla) \vec{u}+\mu \nabla^{2} \vec{\omega}+\nabla \times(\rho \vec{g}) \\
+(\nabla \times \vec{\omega}) \times \nabla \mu+\nabla \times(2 \underline{\dot{\epsilon}} \cdot \nabla \mu) .
\end{aligned}
$$

For 2D cases, the stream function - vorticity formulation [29] may be used, but since it is limited to $2 \mathrm{D}$ cases, it has limited potential for solving engineering problems.

\subsection{The effective properties nanofluid model}

The simplest model used to simulate the flow of a nanofluid is to assume that the suspension of nanoparticles and fluid can be described by effective material properties - viscosity, thermal conductivity, density and specific heat capacity. Several models for these properties have been proposed. 
The model for nanofluid density is the mixing model based on the particle volume fraction $\varphi$. Let us denote the base fluid density as $\rho_{f}$ and the density of solid nanoparticles $\rho_{s}$. In this case, the nanofluid density can be estimated by

$$
\rho_{n f}=(1-\varphi) \rho_{f}+\varphi \rho_{s} .
$$

The apparent viscosity of the nanofluid suspension $\mu_{n f}$ is different from the base fluid viscosity $\mu_{f}$, because of inclusion of nanoparticles. Several approaches aimed at estimating nanofluid viscosity were proposed. Detailed reviews of these approaches were prepared by Mishra et al. [30] and Kumar et al. [31]. The problem was first addressed by Einstein [32] who considered spherical particles at very low concentration $(\varphi<0.02)$. He proposed the following model for nanofluid viscosity

$$
\frac{\mu_{n f}}{\mu_{f}}=(1-2.5 \varphi)
$$

This formula was obtained by considering a dilute dispersion of small rigid spheres. Later, Brinkman [33] corrected the formula, so it is valid for $(\varphi<0.04)$. It reads:

$$
\frac{\mu_{n f}}{\mu_{f}}=(1-\varphi)^{-2.5} .
$$

Many other formulas have been proposed incorporating temperature dependence (Nguyen et al. [34]), using statistical nanomechanics (Avsec and Oblak [35]) or incorporating the volumetric effect of viscosity (Bicerano et al. [36]. An exponential formula was proposed by Cheng and Law [37] and Ward [38]. Experimental studies also yielded many correlations, which considered different influences, for example, studies of particle size and shape (Nguyen et al. [34]) show positive, negative or no dependence on viscosity. The effect of increasing nanoparticle volume concentration (Prasher et al. [39]) was shown to increase viscosity. Goharshadi et al. [40] have shown that when nanoparticle size distribution is broad this leads to less viscous nanofluid as compared to a suspension of uniform particles at the same volume fraction. Chen et al. [41] studied particle aggregation to predict its effect on effective volume fraction. The effect of temperature on viscosity is well known, Andrade equation [42] is usually used. Xian-Ju [43] investigated $\mathrm{pH}$ values, where nanofluid exhibits the least viscosity. Kumar et al. [31] reported that the dispersion method has an effect on the nanofluid viscosity. The effect of shear rate was studied by Abareshi et al. [44] discovering that at high shear rate nanofluids display more Newtonian behaviour. The effect of surfactants was considered by Hung and Chou [45].

Khanafer et al. [46] proposed a mixture model for nanofluid heat capacitance:

$$
\left(\rho c_{p}\right)_{n f}=(1-\varphi)\left(\rho c_{p}\right)_{f}+\varphi\left(\rho c_{p}\right)_{s} .
$$

The same idea can be employed for thermal expansion coefficient: $(\rho \beta)_{n f}=(1-$ $\varphi)(\rho \beta)_{f}+\varphi(\rho \beta)_{s}$. When the definition of $\rho_{n f}$ is taken into account, we can write:

$$
\beta_{n f}=\beta_{f}\left[\frac{1}{1+\frac{(1-\varphi) \rho_{f}}{\varphi \rho_{s}}} \frac{\beta_{s}}{\beta_{f}}+\frac{1}{1+\frac{\varphi}{1-\varphi} \frac{\rho_{s}}{\rho_{f}}}\right] .
$$

The Maxwell-Garnett formula [47], [48] can be used to estimate the effective thermal conductivity of the nanofluid:

$$
k_{n f}=k_{f} \frac{k_{s}+2 k_{f}-2 \varphi\left(k_{f}-k_{s}\right)}{k_{s}+2 k_{f}+\varphi\left(k_{f}-k_{s}\right)} .
$$


This expression assumes that the particles are spherical and it is applicable for small temperature gradients.

\subsection{The mixture model}

The effective properties model assumes that the particle distribution is uniform throughout the whole flow field regardless of the flow conditions and temperature. This assumption is supported by the fact that the particle Stokes number (the ratio of the particle response time scale and the characteristic flow relaxation time scale) is very low

$$
S t=\frac{t_{p}}{t_{f}} \ll 1, \quad t_{p}=\frac{\rho_{p} d_{p}^{2}}{18 \mu},
$$

where $d_{p}$ is the particle diameter. With a very short response time a nanoparticle is able to follow the fluid exactly and due to the huge number of particles involved the assumption of effective properties seems reasonable.

However, experimental studies (Wen and Ding [49]) have shown, that the single-phase effective properties model may not be always applicable. They found that the distribution of particles is non-uniform and that the lower concentration of particles close to the walls leads to lower heat transfer rates. Buongiorno [50] and others [51]-[53] have considered possible reasons for the fact that the particles do not follow the fluid, i.e. that there is slip between the flow and the particle velocity.

Buongiorno [50] considered several mechanisms. In turbulent flow, the Kolmogorov time scale (i.e. the characteristic relaxation time) for the smallest turbulent eddies scales as $R e^{-1 / 2}$ and thus may become comparable to the particle response time. When this happens (at high $R e$ ) the particle will be unable to keep up with the turbulent structures and thus develop slip.

The second mechanism is the Brownian diffusion. Numerous collisions of nanoparticles and the base fluid molecules in random-like motion. If we consider nanoparticles as large fluid molecules with average kinetic energy $1 / 2 k_{B} T$ we can use the Stokes-Einstein equation for Brownian diffusivity

$$
D_{B}=\frac{k_{B} T}{3 \pi \mu d_{p}},
$$

where the Boltzmann constant is denoted by $k_{B}$. The resulting nanoparticle mass flux may be modelled using a Fickian gradient hypothesis yielding

$$
\vec{j}_{B}=-\rho_{p} D_{B} \nabla \varphi
$$

A nanoparticle also exhibits diffusion due to the temperature gradient in the fluid. This effect is called thermophoresis and can be modelled by the introduction of the thermophoretic nanoparticle mass flux

$$
\vec{j}_{T}=-\rho_{p} D_{T} \frac{\nabla T}{T}, \quad D_{T}=\beta \frac{\mu}{\rho_{f}} \varphi,
$$

where $\beta=0.26 k_{f} /\left(2 k_{f}+k_{p}\right)$ [54], [55].

The mixture model considers the nanofluid to be a two-component mixture of nanoparticles and the base fluid. We have identified two diffusive mechanisms, which govern the nanoparticle motion relative to the flow velocity. We may use them to write the nanoparticle concentration transport equation. Realizing that accumulation of nanoparticles in a control volume can be caused by convective or diffusive transport we can write the 
following transport equation

$$
\frac{\partial \varphi}{\partial t}+(\vec{u} \cdot \nabla) \varphi=-\nabla \cdot\left(\vec{j}_{B}+\vec{j}_{T}\right)
$$

Introducing Brownian (14) and thermophoretic (15) mass fluxes we arrive at:

$$
\frac{\partial \varphi}{\partial t}+(\vec{u} \cdot \nabla) \varphi=\nabla \cdot\left(D_{B} \nabla \varphi+D_{T} \frac{\nabla T}{T}\right) .
$$

The energy conservation equation for the nanofluid needs to be adapted to include the added heat flux due to the changing particle concentration:

$$
\rho c_{p}\left(\frac{\partial T}{\partial t}+\vec{u} \cdot \nabla T\right)=-\nabla \cdot\left(-k \nabla T+c_{p} T\left(\vec{j}_{B}+\vec{j}_{T}\right)\right)+c_{p, p} T \nabla \cdot\left(\vec{j}_{B}+\vec{j}_{T}\right),
$$

where the heat capacity of the nanofluid is denoted by $c_{p}, c_{p, p}$ is the heat capacity of particles and $k$ is the thermal conductivity. This simplifies to

$$
\rho c_{p}\left(\frac{\partial T}{\partial t}+\vec{u} \cdot \nabla T\right)=\nabla \cdot(k \nabla T)-c_{p, p}\left(\vec{j}_{B}+\vec{j}_{T}\right) \cdot \nabla T,
$$

where we took $\nabla\left(c_{p, p} T\right)=c_{p, p} \nabla T$ due to the assumption of thermal equilibrium between the fluid and particles. Inserting the Brownian and thermophoretic heat fluxes into the equation gives

$$
\rho c_{p}\left(\frac{\partial T}{\partial t}+\vec{u} \cdot \nabla T\right)=\nabla \cdot(k \nabla T)+\rho_{p} c_{p, p}\left(D_{B} \nabla \varphi \cdot \nabla T+D_{T} \frac{\nabla T \cdot \nabla T}{T}\right) .
$$

\subsection{Multiphase Euler-Lagrange model}

In this approach, we consider numerically following the nanoparticles within the flow field in a Lagrangian manner. Since the particles are very small compared to characteristic flow field length scales, we consider using pointwise approximation and one-way coupling between the continuous fluid phase and particles.

Ravnik et al. [56] have used Euler-Lagrange model. The concentration of nanoparticles is written as $c(\vec{r})=N(\vec{r}) / V(\vec{r})$. Here $\vec{r}$ is a position vector in the flow domain, the number of particles is $N(\vec{r})$ and a small volume at $\vec{r}$ is denoted by $V(\vec{r})$. The particles are very small and the particle concentration in the nanofluid suspension may reach as high as $10 \%$. For this reason, it is computationally too demanding to simulate all particles. The authors proposed that only a computationally feasible a portion of all particles is tracked.

They developed the following expression to calculate the nanoparticle volume fraction

$$
\varphi(\vec{r})=\varphi_{0}+\varphi^{\prime}\left(\frac{c(\vec{r})}{\frac{1}{V} \int_{V} c(\vec{r}) d V}-1\right) .
$$

Here $\varphi^{\prime}$ is the part of volume fraction estimated by tracking individual particles and $\varphi_{0}$ is the bulk volume fraction.

Tracking of particles in a fluid is usually done by solving a system of ordinary differential equations for particle position, velocity, orientation and angular velocity using a Runge-Kutta 
type numerical method. However, in the case of particles with very low Stokes number, we may assume that the particles perfectly follow the fluid streamlines. In order to account for Brownian motion and the thermophoresis effect [57], [54], [58] the particle velocity must be perturbed. For example, [58], suspension of $r_{0}=1 \mathrm{~nm}$ aluminium oxide nanoparticles in water was found to have the following thermophoretic velocity

$$
\vec{u}_{t p}=-1264\left(\frac{r}{r_{0}}\right)^{-1.417} \frac{\mu_{f}}{\rho_{f}} \frac{\nabla T}{T} .
$$

The equation of motion for particles is then: $\vec{r}(t+\Delta t)=\vec{r}(t)+\left(\vec{u}_{f}(\vec{r})+\vec{u}_{t p}\right) \Delta t$, where $\vec{u}_{f}(\vec{r})$ is the fluid velocity at the location of the nanoparticle. Such an approach is computationally more affordable than solving the standard equations of particle motion using Runge-Kutta type ordinary differential equations solvers and thus enable simulation of a higher number of particles alongside the standard CFD simulation.

\subsection{CFD codes and discretization}

Virtually all computational fluid dynamics software packages are capable of simulating flow and heat transfer of nanofluids using the single-phase effective properties approach since the modification of fluid properties is a straightforward task. Other approaches require either additional user-defined algorithms or routines (UDF's in Fluent or User Fortran in CFX) or development of special purpose codes.

The most widely used CFD codes are CFX, Fluent, Star-CCM++ and OpenFOAM [59], which use the finite volume and finite element methods with the governing equations written in primitive variables formulation. Many research groups develop in-house CFD codes [28], [27], [60]-[62] using a wide variety of numerical approaches (e.g. boundary elements, spectral codes, etc.), and an even wider selection of nanofluid modelling methods. The Navier-Stokes equations can alternatively be written in velocity-vorticity formulation [27] or for 2D cases in stream function-vorticity formulation [29].

\section{CONCLUSIONS}

In this paper, we reviewed different approaches to nanofluid modelling showing the advantages and weaknesses of each. The effective properties approach is very easy to implement into existing general purpose CFD codes and thus enables researchers a quick method of nanofluid simulation. When coupled with experimentally determined nanofluid properties it can provide reliable simulation results. However, when used with simplified generic models for nanofluid properties it can lead to less accurate results.

The implementation of the mixture model is more demanding but it can still be used with commercial CFD codes with the use of user-supplied algorithms. Compared to effective properties model, the mixture model takes into account changing nanoparticle concentration due to convective and diffusive transport processes. It also accounts for thermophoresis and Brownian diffusion of nanoparticles.

The Euler-Lagrange models require a dedicated Lagrangian particle tracking solve in addition to the standard CFD code. This approach shows great promise for an accurate description of the underlying physical phenomena, however, it is hindered by the difficulty of accounting for a huge number of nanoparticles present in a typical engineering nanofluid application. 


\section{REFERENCES}

[1] Choi, S.U.S., Enhancing thermal conductivity of fluids with nanoparticles. Develop Appl Non Newtonian Flows, 66, pp. 99-106, 1995.

[2] Yang, Y., Zhang, Z.G., Grulke, E.A., Anderson, W.B. \& Wu, G., Heat transfer properties of nanoparticle-in-fluid dispersions (nanofluids) in laminar flow. Int $J$ Heat Mass Transfer, 48, pp. 1107-1116, 2005.

[3] Minea, A.A., Uncertainties in modeling thermal conductivity of laminar forced convection heat transfer with water alumina nanofluids. Int J Heat Mass Transfer, 68, pp. 78-84, 2014.

[4] Wang, X.Q. \& Mujumdar, A.S., Heat transfer characteristics of nanofluids: a review. Int J Therm Sci, 46, pp. 1-19, 2007.

[5] Oztop, H.F. \& Abu-Nada, E., Natural convection of water-based nanofluids in an inclined enclosure with a heat source. Int J Heat Fluid Flow, 29, pp. 1326-1336, 2008.

[6] Abu-Nada, E. \& Oztop, H.F., Effects of inclination angle on natural convection in enclosures filled with Cu-water nanofluid. Int J Heat Fluid Flow, 30, pp. 669-678, 2009.

[7] Ögüt, E.B., Natural convection of water-based nanofluids in an inclined enclosure with a heat source. Int J Therm Sci, 48, pp. 2063-2073, 2009.

[8] Hwang, K.S., Lee, J.H. \& Jang, S.P., Buoyancy-driven heat transfer of water-based $\mathrm{Al}_{2} \mathrm{O}_{3}$ nanofluids in a rectangular cavity. Int J Heat Mass Transfer, 50, pp. 4003-4010, 2007.

[9] Ho, C.J., Chen, M.W. \& Li, Z.W., Numerical simulation of natural convection of nanofluid in a square enclosure: Effects due to uncertainties of viscosity and thermal conductivity. Int J Heat Mass Transfer, 51, pp. 4506-4516, 2008.

[10] Matin, M.H. \& Pop, I., Natural convection flow and heat transfer in an eccentric annulus filled by Copper nanofluid. Int J Heat Mass Transfer, 61, pp. 353-364, 2013.

[11] Sheikholeslami, M., Gorji-Bandpy, M. \& Vajravelu, K., Lattice Boltzmann simulation of magnetohydrodynamic natural convection heat transfer of $\mathrm{Al}_{2} \mathrm{O}_{3}$ water nanofluid in a horizontal cylindrical enclosure with an inner triangular cylinder. Int $J$ Heat Mass Transfer, 80, pp. 16-25, 2015.

[12] Sheremet, M.A., Pop, I. \& Rahman, M.M., Three-dimensional natural convection in a porous enclosure filled with a nanofluid using Buongiorno's mathematical model. Int $J$ Heat Mass Transfer, 82, pp. 396-405, 2015.

[13] Minea, A.A. \& Lorenzini, G., A numerical study on $\mathrm{ZnO}$ based nanofluids behavior on natural convection. Int J Heat Mass Transfer, 114, pp. 286-296, 2017.

[14] Kim, B.S., Lee, D.S., Ha, M.Y. \& Yoon, H.S., A numerical study of natural convection in a square enclosure with a circular cylinder at different vertical locations. Int J Heat Mass Transfer, 51, pp. 1888-1906, 2008.

[15] Ternik, P., Conduction and convection heat transfer characteristics of water-Au nanofluid in a cubic enclosure with differentially heated side walls. Int J Heat Mass Transfer, 80, pp. 368-375, 2015.

[16] Hu, Y., He, Y., Qi, C., Jiang, B. \& Schlaberg, H.I., Experimental and numerical study of natural convection in a square enclosure filled with nanofluid. Int J Heat Mass Transfer, 78, pp. 380-392, 2014.

[17] Abu-Nada, E., Application of nanofluids for heat transfer enhancement of separated flows encountered in a backward facing step. Int J Heat Fluid Flow, 29(1), pp. 242249, 2008.

[18] Mirmasoumi, S. \& Behzadmehr, A., Effect of nanoparticles mean diameter on mixed convection heat transfer of a nanofluid in a horizontal tube. Int J Heat Fluid Flow, 29, pp. 557-566, 2008. 
[19] Akbarinia, A. \& Behzadmehr, A., Numerical study of laminar mixed convection of a nanofluid in horizontal curved tubes. Appl Therm Eng, 27, pp. 1327-1337, 2007.

[20] Tiwari, R.K. \& Das, M.K., Heat transfer augmentation in a two-sided lid-driven differentially heated square cavity utilizing nanofluids. Int J Heat Mass Transfer, 50, pp. 2002-2018, 2007.

[21] Torii, S., Turbulent heat transfer behavior of nanofluid in a circular tube heated under constant heat flux. Adv Mech Eng, 2010, pp. Article ID 917612, 7 pages, 2010.

[22] Garoosi, F., Bagheri, G. \& Rashidi, M.M., Two phase simulation of natural convection and mixed convection of the nanofluid in a square cavity. Powder Tech, 275, pp. 239256, 2015.

[23] Seyyedi, S.M., Dayyan, M., Soleimani, S. \& Ghasemi, E., Natural convection heat transfer under constant heat flux wall in a nanofluid filled annulus enclosure. Ain Shams Eng J, 6(1), pp. 267-280, 2015.

[24] Abdallaoui, M.E., Hasnaoui, M. \& Amahmid, A., Numerical simulation of natural convection between a decentered triangular heating cylinder and a square outer cylinder filled with a pure fluid or a nanofluid using the lattice Boltzmann method. Powder Tech, 277, pp. 193-205, 2015.

[25] Elshehabey, H.M., Hady, F.M., Ahmed, S.E. \& Mohamed, R.A., Numerical investigation for natural convection of a nanofluid in an inclined L-shaped cavity in the presence of an inclined magnetic field. Int Comm Heat Mass Tran, 57, pp. 228-238, 2014.

[26] Kefayati, G.H.R., FDLBM simulation of entropy generation due to natural convection in an enclosure filled with non-Newtonian nanofluid. Powder Tech, 273, pp. 176-190, 2015.

[27] Ravnik, J., Skerget, L. \& Hribersek, M., Analysis of three-dimensional natural convection of nanofluids by BEM. Eng Anal Bound Elem, 34(12), pp. 1018-1030, 2010.

[28] Ravnik, J., Škerget, L. \& Žunič, Z., Velocity-vorticity formulation for 3D natural convection in an inclined enclosure by BEM. Int J Heat Mass Transfer, 51(17-18), pp. 4517-4527, 2008.

[29] Ramšak, M. \& Škerget, L., A subdomain boundary element method for high-Reynolds laminar flow using stream function - vorticity formulation. Int J Numer Meth Fl, 46, pp. 815-847, 2004.

[30] Mishra, P.C., Mukherjee, S., Nayak, S.K. \& Panda, A., A brief review on viscosity of nanofluids. International Nano Letters, 4(4), pp. 109-120, 2014.

[31] Kumar, P., Kumar, J. \& Suresh, S., Review on nanofluid theoretical viscosity models. IJEIR, 1(2), pp. 182-188, 2012.

[32] Einstein, A., Eine neue Bestimmung der Moleküldimensionen. Annalen der Physik, 324(2), pp. 289-306, 1906.

[33] Brinkman, H.C., The viscosity of concentrated suspensions and solutions. J Chem Phys, 20, pp. 571-581, 1952.

[34] Nguyen, C.T., Desgranges, F., Roy, G., Galanis, N., Mare, T., Boucher, S. \& Angue Mintsa, H., Temperature and particle-size dependent viscosity data for water-based nanofluids - Hysteresis phenomenon. Int J Heat Fluid Flow, 28(6), pp. 1492-1506, 2007.

[35] Avsec, J. \& Oblak, M., The calculation of thermal conductivity, viscosity and thermodynamic properties for nanofluids on the basis of statistical nanomechanics. Int J Heat Mass Transfer, 50(21-22), pp. 4331-4341, 2007.

[36] Bicerano, J., Douglas, J.F. \& Brune, D.A., Model for the viscosity of particle dispersions. J Macromol Sci Polymer Rev, 39(4), pp. 561-642, 1999. 
[37] Cheng, N.S. \& Law, A.W.K., Exponential formula for computing effective viscosity. Powder Tech, 129(1-3), pp. 156-160, 2003.

[38] Ward, A.G., The viscosity of pure liquids. Trans Faraday Soc, 33, p. 88, 1937.

[39] Prasher, R., Song, D., Wang, J. \& Phelan, P., Measurements of nanofluid viscosity and its implications for thermal applications. Appl Phys Lett, 89(13), p. 133108, 2006.

[40] Goharshadi, E.K., Ahmadzadeh, H., Samiee, S. \& Hadadian, M., Nanofluids for heat transfer enhancement-a review. Phys Chem Res, 1, pp. 1-33, 2013.

[41] Chen, H., Witharana, S., Jin, Y., Kim, C. \& Ding, Y., Predicting thermal conductivity of liquid suspensions of nanoparticles (nanofluids) based on rheology. Particuology, 7(2), pp. 151-157, 2009.

[42] Andrade, E.d.C., LVIII. A theory of the viscosity of liquids - Part II. Philos Mag (5), 17(113), pp. 698-732, 1934.

[43] Xian-Ju, W. \& Xin-Fang, L., Influence of $\mathrm{pH}$ on nanofluids' viscosity and thermal conductivity. Chin Phys Soc, 26(5), p. 56601, 2009.

[44] Abareshi, M., Sajjadi, S.H., Zebarjad, S.M. \& Goharshadi, E.K., Fabrication, characterization, and measurement of viscosity of $\alpha$-Fe2O3-glycerol nanofluids. $J \mathrm{Mol}$ Liq, 163(1), pp. 27-32, 2011.

[45] Hung, Y.H. \& Chou, W.C., Chitosan for suspension performance and viscosity of MWCNTs. Inter J Chem Eng Appl, 3(5), pp. 347-353, 2012.

[46] Khanafer, K., Vafai, K. \& Lightstone, M., Buoyancy-driven heat transfer enhancement in a two-dimensional enclosure utilizing nanofluids. Int J Heat Mass Transfer, 46, pp. 3639-3653, 2003.

[47] Maxwell, J.C., A Treatise on Electricity and Magnetism, 2nd ed, Clarendon Press: Oxford, UK, 1881.

[48] Shukla, R.K. \& Dhir, V.K., Numerical study of the effective thermal conductivity of nanofluids. ASME Summer Heat Transfer Conference, 2005.

[49] Wen, D. \& Ding, Y., Experimental investigation into convective heat transfer of nanofluids at the entrance region under laminar flow conditions. Int J Heat Mass Transfer, 47(24), pp. 5181-5188, 2004.

[50] Buongiorno, J., Convective transport in nanofluids. J Heat Tran, 128(3), p. 240, 2006.

[51] Jang, S.P. \& Choi, S.U.S., Role of Brownian motion in the enhanced thermal conductivity of nanofluids. Appl Phys Lett, 84(21), pp. 4316-4318, 2004.

[52] Malvandi, A. \& Ganji, D.D., Brownian motion and thermophoresis effects on slip flow of alumina/water nanofluid inside a circular microchannel in the presence of a magnetic field. Int J Therm Sci, 84, pp. 196-206, 2014.

[53] Keblinski, P., Phillpot, S., Choi, S. \& Eastman, J., Mechanisms of heat flow in suspensions of nano-sized particles (nanofluids). Int J Heat Mass Transfer, 45(4), pp. 855-863, 2002.

[54] McNab, G. \& Meisen, A., Thermophoresis in liquids. J Colloid Interface Sci, 44(2), pp. 339-346, 1973.

[55] Müller-Steinhagen, H., Cooling-water fouling in heat exchangers. Adv Heat Transfer, 33, pp. 415-496, 1999.

[56] Ravnik, J., Skerget, L., Tibaut, J. \& Yeigh, B.W., Solution of energy transport equation with variable material properties by BEM. Int J Comput Methods Exp Meas, 5(3), pp. 337-347, 2017.

[57] Epstein, P., Zur theorie des radiometers. Z Phys, 54, pp. 537-563, 1929.

[58] Michaelides, E.E., Brownian movement and thermophoresis of nanoparticles in liquids. Int J Heat Mass Transfer, 81, pp. 179-187, 2015.

[59] Weller, H.G., Tabor, G., Jasak, H. \& Fureby, C., A tensorial approach to computational 
continuum mechanics using object-oriented techniques. Comput Phys, 12(6), pp. 620631, 1998.

[60] Stajnko, J.K., Ravnik, J. \& Jecl, R., Natural convection in a square cavity filled with a non-Darcy porous medium saturated with nanofluid by the boundary element method. $J$ Porous Media, 20(10), pp. 921-939, 2017.

[61] Ravnik, J. \& Škerget, L., A numerical study of nanofluid natural convection in a cubic enclosure with a circular and an ellipsoidal cylinder. Int J Heat Mass Transfer, 89, pp. 596-605, 2015.

[62] Kramer Stajnko, J., Jecl, R. \& Ravnik, J., Numerical simulation of convective flow in a non-Darcy porous cavity filled with nanofluid. Int J Comput Methods Exp Meas, 4(4), pp. 454-463, 2016. 